\title{
Neoplastic cerebral aneurysm from metastatic lung adenocarcinoma associated with cerebral thrombosis and recurrent subarachnoid haemorrhage
}
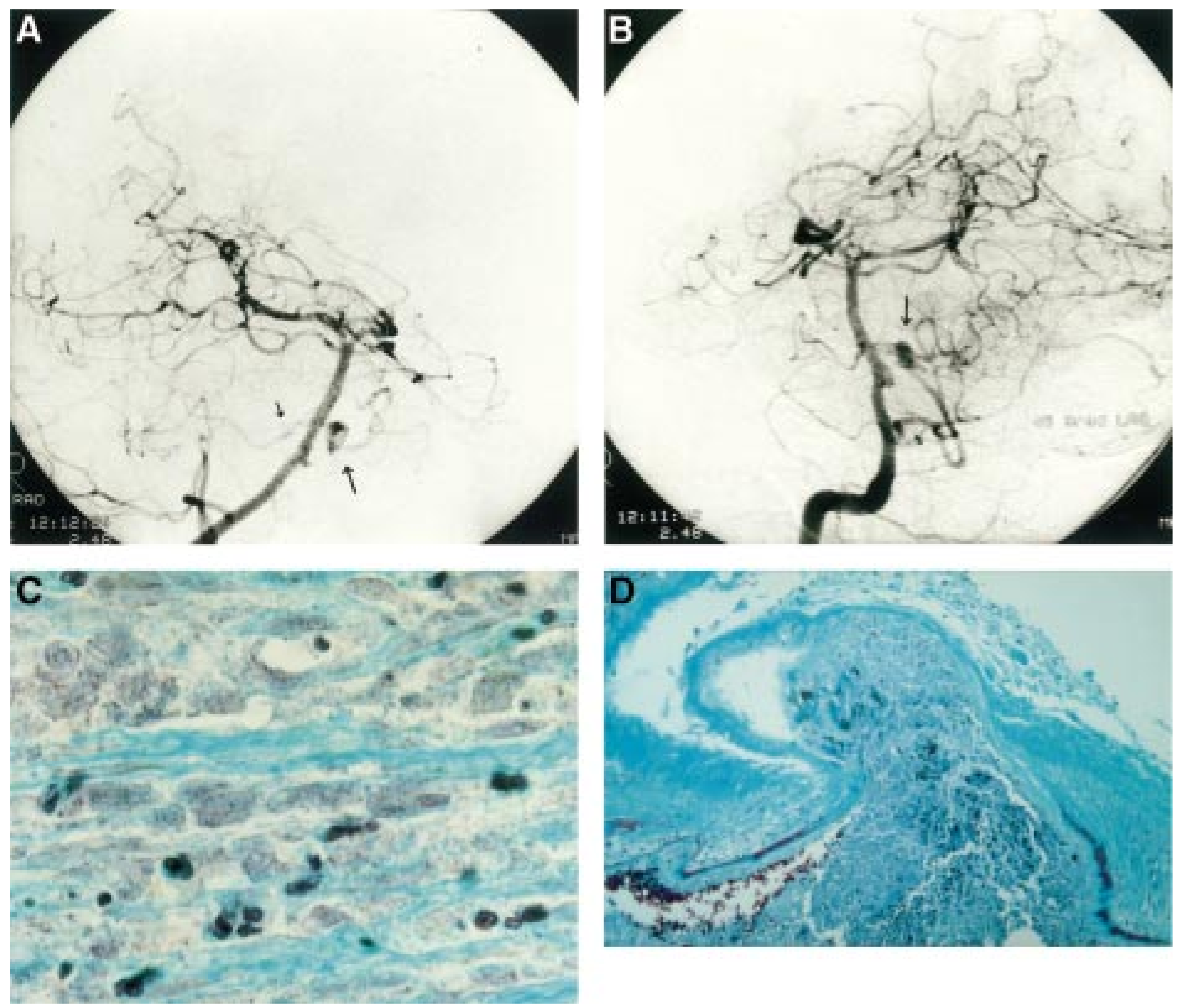

A 38 year old woman presented with a short history of headache, double vision, and rotatory vertigo. Medical history was negative except years of nicotine misuse. Cranial MRI disclosed a large infarction in the area supplied by the left posterior inferior cerebellar artery (PICA).

Occlusion of the left vertebral artery was confirmed by MR angiography.

Five months later, she was again admitted with headache, vomitus, and marked meningism. Cranial CT showed subarachnoid haemorrhage pronounced in the basal cisterns. Cerebral panangiography disclosed no vascular abnormality which could have explained the subarachnoid haemorrhage. Due to the angiography negative subarachnoid haemorrhage 
a repeat panangiography was performed 7 weeks later. Surprisingly, this angiography disclosed a small aneurysm of the proximal left anterior inferior cerebellar artery (AICA).

Due to the fusiform character of the aneurysm, an operation was not performed.

Three weeks later she was again admitted with subarachnoidal rebleeding confirmed by CT. Reangiography showed an enlargement of the established aneurysm (figure A, B; long arrow). An irregularity of the vessel wall was seen on the right AICA (short arrow). A routine chest radiogram and subsequent thoracal CT surprisingly documented a tumour in the upper part of the right lung. A biopsy verified the diagnosis of lung cancer. The clinical condition of the patient deteriorated rapidly during the next few days. She became comatose and died with respiratory paralysis. Before death, a control CT showed a large cerebellar metastatic tumour after administration of contrast medium at the position of the former PICA infarction. Due to the previous infarction it had been masked in CT and MRI.

At postmortem, the wall of the fusiform proximal AICA aneurysm was infiltrated and almost completely destroyed by vital and necrotic adenocarcinoma masses (figure, $C$ ) with histologically proved ruptures as the cause of the recurrent subarachnoid haemorrhage. ${ }^{1-3}$ In the opposite AICA, a developing neoplastic aneurysm was demonstrable with different stages of intramural tumour growth and destruction of the vessel wall (figure, D). ${ }^{4}$ The proximal portion of the basilar artery was covered by an adenocarcinoma cell layer which had caused a thrombus in the left vertebral artery and was the reason for the initial PICA infarction.

J GLIEMROTH

G NOWAK

U KEHLER

H ARNOLD

Department of Neurosurgery

C GAEBEL

Institute of Radiology, Medical University of Lübeck, Lübeck, Germany

Correspondence to: J Gliemroth, MD, Department of Neurosurgery, Medical University of Lübeck, Ratzeburger Allee 160, 23538 Lübeck, Germany.

1 Ho KL. Neoplastic aneurysm and intracranial hemorrhage. Cancer 1982;50:2935-40.

2 Kochi N, Tani E, Yokota M, et al. Neoplastic cerebral aneurysm from lung cancer. 7 Neurosurg 1984;60:640-3.

3 Murata J, Sawamura Y, Takahashi A, et al. Intracerebral hemorrhage caused by a neoplastic aneurysm from small-cell lung carcinoma: case report. Neurosurgery 1993;32:124-6.

4 Hove B, Andersen BB, Christiansen TM. Intracranial oncotic aneurysms from choriocarcinoma. Neuroradiology 1990;32:526-8. 